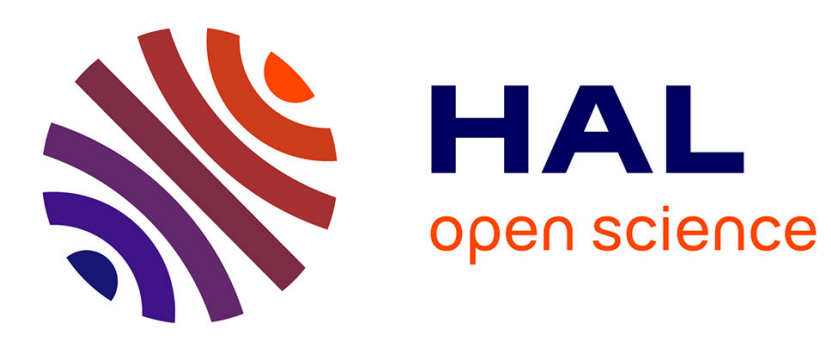

\title{
SOURCE TERMS MODELING OF NUCLEAR REACTORS SPACER GRIDS WITH MIXING VANES FOR CFD SIMULATIONS
}

Sofiane Benhamadouche, Luigi Capone, Yassin A Hassan

\section{- To cite this version:}

Sofiane Benhamadouche, Luigi Capone, Yassin A Hassan. SOURCE TERMS MODELING OF NUCLEAR REACTORS SPACER GRIDS WITH MIXING VANES FOR CFD SIMULATIONS. Computers and Fluids, 2016, 126, pp.141 - 152. 10.1016/j.compfluid.2015.11.011 • hal-01726298

\section{HAL Id: hal-01726298 \\ https://hal.science/hal-01726298}

Submitted on 8 Mar 2018

HAL is a multi-disciplinary open access archive for the deposit and dissemination of scientific research documents, whether they are published or not. The documents may come from teaching and research institutions in France or abroad, or from public or private research centers.
L'archive ouverte pluridisciplinaire HAL, est destinée au dépôt et à la diffusion de documents scientifiques de niveau recherche, publiés ou non, émanant des établissements d'enseignement et de recherche français ou étrangers, des laboratoires publics ou privés. 


\title{
SOURCE TERMS MODELING OF NUCLEAR REACTORS SPACER GRIDS WITH MIXING VANES FOR CFD SIMULATIONS
}

\author{
Luigi Capone $^{1}$,Sofiane Benhamadouche ${ }^{2,4}$, Yassin A. Hassan ${ }^{1,3}$ \\ ${ }^{1}$ Nuclear Engineering Department Texas A\&M University College Station, TX USA \\ ${ }^{2}$ Electricité de France R\&D, MFEE, 6 Quai Watier, 78401 Chatou, France \\ ${ }^{3}$ Mechanical Engineering Department Texas A\&M University College Station, TX USA \\ ${ }^{4}$ LaMSID, UMR CNRS EDF 2832, Clamart, France
}

\begin{abstract}
In the present study, a numerical method was developed to simulate the presence of spacer grids with mixing vanes in nuclear reactors fuel assemblies. These mixing devices usually have a complex morphology that results in difficult mesh procedures and a high computational cost. Spacers and vanes were simulated using momentum sources to reduce the computational power requirements and to enable the feasibility of a quarter of the full reactor core simulations. Several approaches were tested using RANS models. The starting point is calculation with bodyfitted mesh of one grid span of a fuel assembly with spacer grids featuring dimples, springs and mixing vanes. Velocities and Reynolds stresses are extracted from the solution, then converted to source terms. A new computational domain is created with a coarser mesh and without the presence of dimples, springs, and vanes, but source terms are added to the momentum and Reynolds stress transport equations forcing the solution as the detailed geometry computation. The forcing is imposed only in few mesh elements of the domain corresponding to the original location of dimples, spring and vanes. The approach was numerically stable and relatively implemented in the open source code, Code_Saturne (EDF) and in the commercial code Star$\mathrm{ccm}+(\mathrm{Cd}$-Adapco). Best practice was defined and grid sensitivity analysis on different quantities was performed. The robustness of the new numerical method was demonstrated. This new methodology creates an intermediate approach that provides higher spatial resolution than subchannel codes and reduced computational cost compared to detailed CFD simulation. Development of the method was based on anisotropic second order closure models, since lower order models are not able to capture swirling and secondary flow generated by the mixing devices; nevertheless, this novel approach applies also to one and two equations turbulence models, without lack of generality.
\end{abstract}

Keywords: Source terms modeling, mixing vanes, spacer grid, SSG model, Code_Saturne, Star-ccm +

\section{Introduction}

Fuel bundle assemblies are about four meter long in pressurized water reactors. Each assembly is generally composed of a lattice of $17 \times 17$ fuel pins, although some reactors use larger arrays. 
Each assembly is equipped with eight or more spacers with mixing vanes, depending on the vendor. Some designs also include intermediate spacers without mixing vanes. All these devices provide structural stability and ensure heat removal by the coolant. In particular, mixing vanes are added at the top of the spacers to enhance mixing and turbulence generation in the flow to increase the efficiency of heat transfer mechanisms. For even a quarter of core simulation, there is not enough computing power available if all the spacers and mixing devices are considered through a body-fitted mesh. Even the simulation of a single full length assembly 17x17 would require several billion of cells for a LES (Large Eddy Simulation) using wall functions at operational Reynolds numbers of 500000.

Because of these difficulties, there has been great interest in the possibility of simulating fuel bundles of different reactor concepts using CFD, while reducing the mesh size and the computational cost. The main idea is to find an intermediate approach between sub-channels codes and detailed CFD calculations with body fitted meshes. Sub-channel codes provide only limited space dependent information and rely on empirical coefficients derived from experimental data fitting to model the inter-channel exchange of momentum. The assembly problem is characterized by the presence of repeating identical elements like the spacers and vanes. Many researchers have shifted their focus to finding a procedure that allows simulation of a single spacer with detailed CFD and then applying this information to simulate an entire assembly.

A few studies were focused on the implementation of porous media, coarse grid approaches or momentum sources. One of the first studies applied to the nuclear reactor field was conducted by Chandesris et al. (2006), who developed a turbulence model from a porous media volumeaveraged equation and a further developed k- $\varepsilon$ macroscopic model investigated by Nakayama etal. (1999). Chandesris applied the porous media approach to a $5 \times 5$ bundle with spacer grid simulation. Although the model was only for the longitudinal component of the velocity, a comparison between the kinetic turbulence energy decay downstream from the spacer predicted by the model was in good agreement with experimental data. A different approach was implemented by Himmel (2009) and Viellieber et al. (2012). They developed a coarse grid approach for spacers without mixing vanes and wire wraps. The proposed technique extracts volume forces from a fine CFD-detailed simulation made with a body-fitted mesh in the regions of the computational domain where there are objects with a complex shape. Then, a coarse grid is generated which does not account for these complex geometrical features. A comparative algorithm defines the regions that need to be modeled by volumetric sources, as well as how many cells of the detailed mesh correspond to each coarse mesh cell. With this technique, volumetric forces carrying the information about the presence of the complex geometrical features are calculated with a spatial average of the different velocity components over the volumes of all the mesh cells of the detailed simulation. These averaged quantities are then included in each of corresponding coarse mesh cells. An additional hypothesis is that the volumetric forces include the information about friction and turbulence generated in the fine 
calculation. Therefore, Euler equations are solved with body forces on the coarse grid. An improvement of this method was developed by Viellieber, who added an anisotropic porous media to the previous algorithm to match the pressure drop across the spacer. The study presents interesting results for heat transfer calculation, but a detailed analysis of the turbulent quantities is missing. Additionally, the spacer analyzed in that study was not equipped with mixing vanes. Another study was conducted by $\mathrm{Hu}$ et al. (2011) on wire wrap configurations for liquid metal cooled reactors. The wire wrap was modeled with momentum sources imposed in the momentum equation in the same position where the wire was positioned in a body-fitted mesh. The three components of the momentum sources were imposed using formulas related to the friction coefficient, incidence angle of the wire, and velocity. The advantage of this method is that a previous, detailed calculation of full geometry is not needed. Also the other problem is that to impose the momentum sources a mesh has to be created as the wire rap was present in the geometry to reconstruct a smooth position of the cells to impose the sources. This limitation does not reduce the meshing effort for an entire core or a full assembly. A new solution was developed in the present study using a source term modeling techniques. The idea was to generate a solution with a body-fitted mesh for a single grid span using anisotropic turbulence models as SSG (Speziale, Sarkar, Gatsky) Speziale et al. (1991). From this calculation, source terms were extracted in the few volumes containing dimples and springs for the spacer and mixing vanes and then defined for momentum and energy transport equations. The numerical method developed was studied for spacer grid with mixing vanes and implemented in Code_Saturne (2010) and Star-ccm+ (2011).

\section{Source terms modeling technique}

\subsection{Background}

The technique developed in the present study merges concepts coming from two different research areas of computational techniques in fluid dynamics: the immersed boundary method techniques (IBM) and the hybrid method for RANS (Reynolds Averaged Navier Stokes) and LES (Large Eddy Simulations) coupling. The idea of defining the presence of a body in the fluid domain without the use of a body-fitted mesh is one of the main characteristics of the IBM Peskin (1972), (1977). The method literature presents several solutions to model the presence of a wall boundary using body forces with Navier-Stokes or Lattice Boltzmann equations. In this context, the procedure of imposing the presence of a wall with body forces in the flow governing equations is called direct-forcing. There are different ways to reconstruct and impose the body forces with both diffuse interface Mohd-Yusof (1997) and sharp interface schemes Faldum (2000). Additionally, the forcing can be imposed with implicit or explicit methods, depending on the time scheme. 
Kang et al. (2010), (2011) compares several forcing methods to define the presence of a solid boundary using direct forcing method with a diffuse interface scheme. The main idea is to build a Cartesian mesh and model the presence of bodies using interpolating functions which impose the boundary shapes. The presence of the wall is the forced-through source terms in the momentum equations that impose the non-slip condition on the immersed boundary. This technique has many advantages especially for moving objects with large displacements. But when it comes to complex geometries such as spacer grids and fuel bundles, the difficult task of generating a high quality body-fitted mesh is accomplished instead by implementing a complex algorithm for interpolation functions able to reproduce the immersed boundary of the object. Also, the Cartesian mesh needs high refinement to properly represent the interpolating functions the geometry.

The idea of using source terms to force a coarser calculation to resolve a domain featuring complex geometries was derived from the immersed boundary technique. Because source values are generated from previous calculations in this present study, the source can be defined as explicitly imposed. Hybrid methods to couple RANS and LES models are defined to solve part of the domain using RANS and another part with LES. For example, RANS calculations are applied close to the wall and the central part of the domain is solved with LES, which reduces the computational cost of pure LES simulation in resolving the boundary layer Benhamadouche et al. (2008).

It is also possible to separate the domain into two volumes and apply different turbulence models to solve each part. For such an application, LES are used only in the regions with highly unsteady flows. Clearly, the domain between the two regions is the function of interface between the two models, and coupling techniques are needed for the two different sets of equations Frohlich et al. (2008), Keating et al. (2006). The works of Benhamadouche and Keating, describe a coupling of the LES and RANS solution in the interface region using body forces. In fact, if the exchange of information at the interface is processed at the cell centers, a source term is added to the LES equations with the expression (1):

$$
S_{i}=\rho \frac{{\overline{U_{i}}}^{R A N S}-U_{i}^{L E S}}{\tau} \quad i=1,2,3
$$

The instantaneous LES velocity component is coupled at the interface with the average RANS component through a time coupling constant. In Keating's work, a similar expression is used but the force magnitude is multiplied by a weight function that is related to Reynolds stress fluctuations at the interface. In this study, a similar expression to (1) was used to impose the source terms in the momentum and Reynolds stresses transport equations.

\subsection{The numerical approach}


The method used in this study was defined for RANS calculations. The procedure starts with the calculation of the full domain using a body-fitted mesh. In this work, a $5 \times 5$ rod bundle configuration was analyzed. The computational domain extends for one grid span, the spacer is characterized by the presence of two levels of springs and dimples in the middle of them, and the mixing vanes are split type with weld nugget. The calculation was performed using the SSG turbulence model. The SSG model was adopted because it can predict complex rotating flows, such as the ones generated by mixing vanes. Also, SSG models all the Reynolds Stresses using a non-linear return to isotropy term in the pressure rate of strain equations (2) using coefficients in Table 1.

$$
\frac{R_{i j}}{\varepsilon}=\sum_{n=1}^{8} C^{(n)} \mathrm{T}_{i j}^{n} \quad \mathrm{~T}_{i j}^{(2)}=b_{i j}^{2}-\frac{1}{3} b_{k k}^{2} \delta_{i j}
$$

\section{Table1.}

Pressure rate of strain tensor coefficients for SSG model

Once the full geometry computation is obtained, another computational domain has to be generated without the complex features of the spacer. This new domain eliminated vanes, springs and dimples, but the spacer bare walls were kept, as shown in Fig. 1 (bare grid domain).

Fig $1 \boldsymbol{a}, \boldsymbol{b}$. From the left side and top view of the rod bundle and bare walls spacer. All the complex geometrical features were removed. 
Next, the presence of all these elements is replaced by source terms modeling is based on velocities and stresses calculated in the full geometry computation. There is a difference between the positions of the cell centers of mass in the coarser mesh and the one in the full geometry computation. The velocity and Reynolds stresses information need to be extracted by the latter in the volumes specified in Fig. 2:

1. Vanes

2. Spring A

3. Dimples

4. Spring B

For this spacer grid design, the total volume used to extract sources information is less than 5\% of the total domain size. The cells' center of mass positions and the number of cells in the corresponding extraction volumes are different between the full geometry domain and the bare grid domain.

Fig 2. Four volumes used for solution extraction for body fitted mesh calculation and source forcing for bare grid ones.

Using spatial gradients for velocity and Reynolds stresses, all the different quantities extracted are extrapolated to the new positions in Fig. 2 through the gradient formula (3a)for velocities and (3b) for Reynolds stresses:

$$
\begin{aligned}
& \left.u_{i}\right|_{p 1}=\left.u_{i}\right|_{p 0}+\left.\left(\nabla u_{i}\right)\right|_{p 0}\left(\underline{x}_{p 1}-\underline{x}_{p 0}\right) \\
& \left.u_{i} u_{j}\right|_{p 1}=\left.u_{i} u_{j}\right|_{p 0}+\left.\left(\nabla u_{i} u_{j}\right)\right|_{p 0}\left(\underline{x}_{p 1}-\underline{x}_{p 0}\right)
\end{aligned}
$$

This arrangement retains much more information than a spatial average of each quantity, as described in Fig. 3. In fact, the extrapolation generates a new value of different location as it was part of the original solution with linear approximation.

Fig 3. Comparison between the extrapolation and averaging technique to definition velocities and Reynolds stresses in the coarse bare grid domain

This technique is also used in hybrid RANS-LES. Both the Navier Stokes and Reynolds stress transport equations can be written with an addition of a source term, as shown in Equations 4 and 
5, respectively.. The source terms had to be defined as (6), (7) in similar way to the RANS-LES techniques.

$$
\begin{gathered}
\partial_{t} u_{j}+u_{j} \partial_{j} u_{i}=-\partial_{i} p+\frac{1}{\operatorname{Re}} \partial_{j}^{2} u_{i}+S_{i} \text { (4) } \\
\bar{D}_{t}\left\langle u_{i} u_{j}\right\rangle+\partial_{k} T_{k i j}=P_{i j}+R_{i j}-\varepsilon_{i j}+S_{i j}(5) \\
S_{i}=\frac{u_{i}^{\text {forcing }}-u_{i}}{\tau_{\text {coupling }}}
\end{gathered}
$$

The time constant $\tau$ is usually defined as one hundred times the simulation time step, but for the present work it depends on the numerical scheme used to solve the source terms in the equations and is analyzed in the following sections. The source terms are defined for the use of SSG model on the bare grid domain, but the SSG derived sources problem to be solved using different RANS turbulent models as $\mathrm{k}-\varepsilon$ and $\mathrm{k}-\omega-\mathrm{SST}$ as indicated in the following formula to define a source term in the kinetic turbulent equation (8):

$$
S^{\text {tke }}=\frac{k^{\text {forcing }}-k}{\tau_{\text {coupling }}}
$$

\subsection{Implementation in CFD codes}

The present research activity aims to compare the implementation of the method and the computational results of two different CFD codes. The first one is Code_Saturne developed by EDF R\&D Archambeau et al. (2004). It is an in-house open source code and it is based on an unstructured, collocated finite volume solver. The pressure velocity coupling is obtained through SIMPLEC algorithm and the Rhie and Chow interpolation is implemented to avoid odd-even decoupling on structured meshes. The Code_Saturne solver collocates the all the variables at the center of mass of the cells. Several RANS and LES turbulence models are available in this code. As a best practice, a second order centered scheme in space and time is used and a full central scheme is used for the convective term. Non-orthogonal grid cells are implicitly treated by the velocity pressure solver for the gradients reconstruction. Several validations of the code were performed for complex geometries by Benhamadouche et al. (2003). In particular, an LES study was done by Benhamadouche et al. (2009 'a'), (2009 'b') on fuel bundle assemblies using a fully hexahedral mesh and a periodic configuration with four sub-channels. The spacer used for the simulation was equipped with springs, dimples and split-type mixing vanes. As an open source license, the user is allowed to modify the source code. This enabled the testing of two different schemes for the source forcing techniques. There are dedicated subroutines for the implementation of source terms in the momentum and turbulent energy equations depending on 
the turbulence model. The source terms can be treated implicitly or explicitly by the code. Both schemes were tested in the present study, resulting in interesting results. The formulation of the source term is formalized in Equation 9 and Equation 10 with implicit and explicit discretization schemes, respectively:

$$
\begin{gathered}
\frac{\underline{u}^{k+1}-\underline{u}^{k}}{\Delta t}+A \underline{u}^{k+1}+\frac{\underline{u}^{k+1}}{\tau_{\text {coupling }}}=\frac{\underline{u}^{\text {forcing }}}{\tau_{\text {coupling }}} \\
\frac{\underline{u}^{k+1}-\underline{u}^{k}}{\Delta t}+A \underline{u}^{k+1}=\frac{\underline{u}^{\text {forcing }}-\underline{u}^{k}}{\tau_{\text {coupling }}}(10)
\end{gathered}
$$

Pre-calculated quantities were called imposed values and the other the variable ones. The numerical algorithm starts with the initialization of the domain and the allocation of the source term in the defined four volumes at the cells center of mass. All this information is imported from a user defined table. Then the solver forces the solution in the volumes where the forcing is active to couple with source values for the explicit scheme and to be exactly the same as the source tem with the implicit treatment. More details are addressed in the simulations section.

Star-ccm+ is a Cd-Adapco CFD commercial code. It is based on finite volumes schemes and presents several turbulence models. There are two solvers available; coupled and segregated flow. The code is also based on collocated variable arrangement Rhie-and-Chow type pressurevelocity coupling combined with a SIMPLE-type algorithm. Validation for Star-ccm+ in complex industrial cases was broadly studied and there are some works about RANS calculations for fuel bundles with spacer grids Conner et al. (2010). This work is focused on the SSG model, which is unstable in the code even if second-order up-winding is used by default for the convective term. All other RANS, LES, DES (Dethatched Eddy Simulations Shur et al. (2008)) models are stable and reliable in Star-ccm+.

The SSG instability in Star-ccm+ plus the ability to directly code the extrapolation process led to the utilization of Code_Saturne for the full geomrety computation. Star-ccm+ presents options for momentum and turbulent energy equation source options. Using filed function and extrapolated data tables from Code_Saturne, the implementation for the source terms was relatively straight-forward. The only limitation was the implicit scheme implementation for sources due to the impossibility of directly accessing the code routines. Using the default option and the user manual, only the explicit scheme was available. Although a user code subroutine could have overcome this restriction, there is not enough information in the manual for the specific implementation needed in this case. The present scheme was implemented in a commercial code to show the possible application to any kind of CFD solver where source terms option is available. 


\section{Simulations}

Code_Saturne SSG model was used to perform the full geometry calculation with $5 \times 5$ configurations using a fully hexahedral mesh built with Icem-CFD (2009). The simulation was run for 20 flows through with a time step of $10^{-5} \mathrm{~s}$. The computational domain is $32.9 \mathrm{~cm}$ long and the spacer grid is housed in a square channel with $6.61 \mathrm{~cm}$ edge length. The total mesh size was 20 million elements. The Reynolds number was $\mathrm{Re}=100,000$ and the hydraulic diameter $1.178 \mathrm{~cm}$. The $\mathrm{U}_{\text {bulk }}=6.7968 \mathrm{~m} / \mathrm{s}$. Water properties were imposed at $30^{\circ} \mathrm{C}$ temperature. The mesh size was adapted to the Reynolds number and wall functions used. From the simulation data, the velocity components and the Reynolds stresses were extrapolated with gradients formula using a special subroutine available in Code_Saturne. Then three new meshes with different refinements were generated in Icem-CFD using the bare grid showed in Fig. 1. The mesh dimensions are reported in Table 2 .

\section{Table 2}

Bare grid meshes for source forcing technique

Different source forcing options were tested mainly using Code_Saturne and Star-ccm+. But Code_Saturne was mainly used to find a best practice method since the source code was accessible. The different forcing techniques implemented are summarized in Table 3:

Table 3

Different forcing options analyzed with Code_Saturne ${ }^{1}$ :

${ }^{1}$ (" $x$ " indicates enabled and "|" disabled)

All different options were simulated with each mesh. Star-ccm + was tested with the same options for the forcing, but the variable terms in the forcing expressions were not solved implicitly. The difference between implicit and explicit schemes stands in the way the variable term in the source expression is treated by the solver. In the implicit scheme, the variable term is directly added to the matrix diagonal during the solving procedure. The same procedure is applied to Navier Stokes equations and Reynolds stresses. As a consequence of the implicit forcing, the time constant could be imposed as an infinite time that basically fixes the value of the pre-calculated velocity in the cells with source forcing. With the explicit forcing, the coupling procedure with a time constant one hundred times the value of the time step is not able 
to fix the pre-calculated values in the select volumes. This difference in the schemes is demonstrated in the following pictures Fig. 4 and 5. Clearly, it is possible to fix the velocities and stress components in the corresponding positions of objects such as mixing vanes, dimples, or springs by imposing the exact value of the pre-calculated solution as a volumetric condition. Instead, with the explicit forcing shown in Fig. 5, the shadow of the walls imposed by the percolated solution is not visible. Even if the time coupling constant is increased with the explicit scheme, there are slight improvements until a certain value, above which the solver may reach instability. However, the simulations with Code_Saturne were done using a time constant of $10^{-8}$ and the time constant for Star_ccm+ was set to $5.10-4 \mathrm{~s}$ for all calculations which is one hundred times the simulation time step. From previous pictures, it is possible to affirm that the implicit forcing is closer from a theoretical point of view to the IBM methodology and the explicit forcing is similar to a RANS LES hybrid model coupling. In the present case, it can be said it is a pre-calculated RANS to RANS coupling.

Fig. 4. Left: cross sectional view of the bare grid computational domain at the spring A axial position; around each pin the shadow generated by the forcing solution as the springs were represented in the mesh. Right: cross sectional view of the body-fitted mesh where the presence of springs is clearly visible

Fig. 5. Left: cross sectional view of the bare grid computational domain at the mixing vanes axial position; around each pin the shadow generated by the forcing solution as the vanes were represented in the mesh. Right: cross sectional view of the body-fitted mesh where the presence of mixing vanes is clearly visible 
Some options were unstable in Star- $\mathrm{cm}+$, in particular the one with forcing of the axial velocity as described in Table 4. The results section explains that this is not a weakness of the code as the axial velocity forcing does not produce good results for certain variables.

\title{
Table4
}

\author{
Different forcing options analyzed with Star-ccm $+^{2}$ \\ 2 ("x" indicates enabled and "l" disabled)
}

All simulations performed with Code_Saturne were computed using central scheme for the convective term. However, Star-ccm+ didn't allow the use of the central scheme since only $1^{\text {st }}$ and $2^{\text {nd }}$ order up-wind were available, so the latter one was used. Also the gradient reconstruction, the option to use secondary gradients with the least-squared method was turned off for both codes. This minimized the numerical diffusivity because the bare grid meshes are highly orthogonal and have small skewness angles below $45^{\circ}$. As a consequence, the meshes are highly numerically stable and present high convergence in tests performed at the same Reynolds number without any forcing. This condition was essential to estimate the level of numerical stability for all the different forcing options exhibiting very fast convergence. The instability of the axial velocity forcing coupled with the SSG model in Star-ccm+ may be un-related to sources forcing, but further analysis is needed to clarify this issue.

\section{Results}

Several quantities were analyzed to determine which approach was predicting the closest values to the body-fitted mesh calculation. A quantitative comparison between different integral quantities was made: pressure (11), normal components of Reynolds stresses (12)-(13)-(14), kinetic turbulence energy (15) and circulation (16) were spatial averaged over planes corresponding to each cell layer close in the axial direction. In the last part, a qualitative comparison was made related to the structures generated downstream the mixing vanes in the fluid flow.

$$
\begin{gathered}
\langle p\rangle=\frac{1}{A} \iint p d A(11),\left\langle\bar{u}^{2}\right\rangle=\frac{1}{A} \iint \bar{u}^{2} d A(12),\left\langle\bar{v}^{2}\right\rangle=\frac{1}{A} \iint \bar{v}^{2} d A(13),\left\langle\bar{w}^{2}\right\rangle=\frac{1}{A} \iint \bar{w}^{2} d A \\
\langle k\rangle=\frac{1}{A} \iint \frac{1}{2}\left(\bar{u}^{2}+\bar{v}^{2}+\bar{w}^{2}\right) d A \quad(15), \quad \text { Circulation }=\frac{1}{A} \iint \sqrt{\bar{U}^{2}+\bar{V}^{2}} d A
\end{gathered}
$$

This set of calculations was performed with Code_Saturne using implicit forcing. The first comparison was made between the pure forcing of the velocity components. In the first case only, the two tangential velocities were imposed and in the second, axial velocities were imposed 
as well, as demonstrated in Fig. 6-7. The main difference between the two solutions is related to the pressure results. The pressure drop is just slightly underestimated by forcing the two transversal components. This is reasonable since the forcing of the solution and the coarsening of the meshes is not able to reproduce the same blockage ratio generated by the full geometry spacer. It is clear in Fig. 7 that there exists the complete disagreement between the pressure profiles predicted by all three meshes when the axial velocity component is added to the source forcing. However, both approaches result in a reliable prediction of the average circulation. The turbulent quantities were over-predicted by both approaches; in particular the axial velocity forcing generates a higher over-estimation of the three normal Reynolds stress components. The difference between the solutions obtained from forcing and the body-fitted one reached the maximum across the grid. The flow is subject to sharp spatial variation of the turbulent quantities across the grid, therefore without a forcing of the Reynolds stresses; even the pure forcing of the tangential components is not able to reconstruct the correct decay of turbulent kinetic energy. Therefore, from this first analysis, it can be concluded that a forcing of the Reynolds stresses is needed and the forcing of the axial velocity component must be avoided to achieve the closest prediction of the body-fitted mesh solution through source forcing.

Fig. 6 a,b,c,d,e,f. Integral quantities evolution along the axial direction with forcing of tangentials velocity components using SSG model using implicit forcing

Fig. 7 a,b,c,d,e,f. Integral quantities evolution along the axial direction with forcing of tangentials and axial velocity components using SSG model using implicit forcing 
A second comparison was made between the same velocity component forcing options with the addition of the Reynolds stress forcing, as shown in Fig. 8 and 9. The option with tangential velocity components and stresses forcing returned promising results. The turbulent quantities shows a very good agreement with the body-fitted mesh solution, and both the circulation and the pressure drop are basically the same as the previous case without stress forcing. It seems that the velocity forcing is partially able to reconstruct the turbulence decay along the channel through the grid. The stress forcing refines the turbulent energy reconstruction, providing a better definition of the turbulence generated from the grid. It has to be stated that the Reynolds stresses are second order quantitites compared to velocity components. Therefore, in this case, the forcing is at both first and second order conditions. Additionally, a huge improvement in the Reynolds stress predictions appeared in the case when the axial velocity component forcing, but the wrong estimation of the pressure profile was also present with the stress forcing. It is clear that the best method seems to be the forcing of tangential velocity components and Reynolds stresses. Also it has to be mentioned that all the tangential quatieties such as circulation and u-u, $\mathrm{v}-\mathrm{v}$ stress components are well predicted. These quantites are of foundamental imporatance in determining the correct physics generated by mixing vanes through the swirl enhanchment. The three meshes provide very similar integral quantities, even at the coarsest refinement. In particoular, the one million mesh per grid span seems a worthy compromise for multiple assemby simulations. Although the present method is benchmarked with body-fitted mesh calculation, it is intended to improve the spatial resolution and fidelity simulations forsubchannel codes, a goal which would be achieved using even the coarsest mesh.

Using Code_Saturne, the best forcing combination was established and the same methods were tested in Star-ccm+ using the explicit formulation for the forcing. The results are reported only for the best practice case with tangential velocities and Reynolds stresses forcing in Fig. 10. The results were interesting because the explicit forcing demonstrated the inability to correctly reproduce turbulent quantities. The circulation and pressure drop are predicted with similar results to the implicit scheme. However, Reynolds stresses and turbulent kinetic energy showed completely different behaviour, especially upstream of the spacer grid. Several sensistivity test were conducted by tuning the turbulence intensity at the inlet or modifying the coupling time constant. In the case of explicit coupling, the decrease of the coupling time constant can generate instability in the solver beyond a certain threshold. None of the previous modifications improved the result. Thus, it is evident that a forcing defined in Equation 10 seems to be effective only for velocities and pressure. The Reynolds stresses transport equations need a very precise volumetric boundary condition to regenerate the same turbulent field as the body-fitted caculation. Therefore, this can be achieved only through the implicit coupling, which allow the use of infinitesimal coupling constant and exact imposition of the value of the stresses in the exatrapolated positions contained in the forcing volumes. 
Fig. 8 a,b,c,d,e,f. Integral quantities evolution along the axial direction with forcing of tangentials velocity components and Reynolds stresses using SSG model using implicit forcing

Fig. 9 a,b,c,d,e,f. Integral quantities evolution along the axial direction with forcing of tangentials and axial velocity components and Reynolds stresses using SSG model using implicit forcing

Fig. 10 a,b,c,d,e,f. Integral quantities evolution along the axial direction with forcing of tangentials components and Reynolds stresses using SSG model using explicit forcing

A qualitative comparison was also made to understand if some of the turbulent structures generated by the mixing vanes were regenerated locally by the forcing as a function of refinement, since only integral quantities were compared. A typical characteristic of split type vanes is the generation of complex recirculation structures at few hydraulic diameters downstream the vanes. Therefore some cross sectional plane were reported to compare the flow features of the body-fitted solution and the source forcing with tangential velocities and Reynolds stresses, as described in Fig. 11 through 14. 
Fig. 11 Left: Body fitted solution Right: 6 million mesh tangential velocity structures $1.5 \mathrm{Dh}$ downstream mixing vanes

Fig. 12 Left: 20,000 elements mesh Right: 1 million mesh tangential velocity structures 1.5 Dh downstream mixing vanes

Fig. 13 Left: Body fitted solution Right: 6 million mesh tangential velocity structures $5 \mathrm{Dh}$ downstream mixing vanes

Fig. 14 Left: 200000 elements mesh Right: 1 million mesh tangential velocity structures 5 Dh downstream mixing vanes

The presence of complex structures is evident in all meshes refinements and at both axial locations. In particular, at $1.5 \mathrm{Dh}$ downstream the mixing vanes, the generation of elliptical shaped vortices in each sub-channel is present even in the coarsest mesh. Even at 5Dh, all meshes capture the transition of the elliptical vortices to circular ones and the odd-even counter rotation due to the different orientation of the vanes between two consecutive sub-channels. In Fig. 15 it was reported a preliminary calculation using the implicit forcing method for four grid spans. The total size of the mesh was four million elements and the same sources values were used to for each spacer. 
Fig. 15 Calculation with SSG model for four grid spans with a total mesh size of four million elements

\section{Conclusion}

A new computational method was implemented to simulate complex, repeating geometries for industrial cases. The main objective of this work was the assessment of the feasibility of a forcing technique that was able to reproduce the presence of solid structures in the flow using momentum term forcing. A detailed analysis of integral turbulence quantities, pressure and circulation was developed for testing different forcing approaches. The most promising one resulted in the forcing of tangential velocity components and Reynolds stresses. These could be defined as forcing of first and second order quantities. There was a certain discrepancy with the predicted pressure drop across the mixing grid, but this problem could be easily overcome using a volumetric pressure drop tensor based on the body-fitted mesh solution. Additionally, the implicit algorithm for the source term is the most reliable approach for all quantities compared to the explicit one. In future work, the techniques have to be benchmarked with experimental data and the thermal transport problem should be investigated.

\section{Acknowledgments}

This research was developed with founding from EPRI (Electric Power Research Institute) and EDF R\&D.

The authors acknowledge the EDF R\&D Chatou and Texas A\&M Supercomputing Facility (http://sc.tamu.edu/) for providing computing resources useful in conducting the research reported in this paper.

\section{References}

Archambeau, F., Mechitoua, N., Sakiz, M., 2004. Code_Saturne: a finite volume code for the computation of turbulent incompressible flows: industrial applications. International Journal on Finite Volumes (1), 1 .

Benahmadouche, S., Laurence, D., 2003. LES Coarse LES and transient RANS comparison on flow across tube bundle. International Journal of Heat and Fluid Flow (24) 470-479. 
Benhamadouche, S., Fournier, Y., Billard, F., Jarrin, N., Prosser, R., 2008. RANS/LES coupling in the industrial CFD tool Code_Saturne: implementation and first results. Turbulence, Heat and Mass Transfer.

Benhamadouce, S., Le-Maitre, C., 2009 'a'. Large eddy simulation of the flow along four subchannels downstream a mixing grid in a PWR. NURETH-13 Kanazawa City Japan.

Benhamadouche, S., Moussou, P., Le-Maitre, C., 2009 'b'. CFD estimation of the flow-induced vibrations of the fuel rod downstream a mixing-grid. Proceedings of PVPASME Prague Czech Republic.

Chandesris, M.,Serre, G., Sagaut, P., 2006. A macroscopic turbulence model for flow in porous media suited for channel, pipe and rod bundle flows. International Journal of Heat and Mass Transfer (49) 2739-2750.

Code_ Saturne User Manual Version RC-2. 2010 EDF R\&D.

Conner, M.E., Baglietto, E., Elmahdi, A.M., 2010. CFD methodology and validation for single-phase flow in PWR fuel assemblies. Nuclear Engineering and Design (240), 2088-2095.

Fadlun, E.A., Verzicco, R., Orlandi P., Mohd-Yusof, J., 2000. Combined immersed-boundary finite-difference methods for three-dimensional complex flow simulations. Journal of Computational Physics (161), 35-60.

Frohlich, J.,Von Terzi, D., 2008. Hybrid LES/RANS methods for the simulation of turbulent flows. Progress in Aerospace (44), 349-377.

Himmel, S. P., 2009. Modellierung des strömungsverhaltens in einem HPLWR-brennelement mit drahtwendelabstandshaltern. FZKA 7468.

Hu, R., Fanning, T. H., 2011. Development of a three-dimensional momentum source model for wire-wrapped rod bundles. NURETH-14 Toronto, Ontario Canada.

Icem-CFD Manual v 12.0, 2009. Ansys.

Kang, S. K., Hassan, Y. A., 2010. A comparative study of direct-forcing immersed boundarylattice Boltzmann methods for stationary complex boundaries. International Journal of Numerical Methods in Fluids (66), 1132-1158.

Kang, S. K., Hassan,Y. A., 2011. A direct-forcing immersed boundary method for the thermal lattice Boltzmann method. Computers \& Fluids (49), 36-45.

Keating, A., De Prisco, G., Piomelli, U., 2006. Interface conditions for hybrid RANS/LES calculations. International Journal of Heat and Fluid Flow 27, 777-788. 
Mohd-Yusof, J., 1997. Combined immersed boundaries B-spline methods for simulations of flows in complex geomatries. CTR Annual Research briefs, NASA Ames/Stanford University, 317-327.

Nakayama, A., Kuwahara, F., 1999. A macroscopic turbulence model for flow in a porous medium. Journal of Fluids Engineering 121, 427-433.

Peskin, C.S., 1972. Flow patterns around heart valves: a numerical method. Journal of Computational Physics 10, 251-271.

Peskin, C.S., 1977. Numerical analysis of the blood flow in the heart. Journal of Computational Physics 25, 220-252.

Speziale, G. S., Sarkar, S., Gatski, T. B., 1991. Modelling the pressure-strain correlation of turbulence: an invariant dynamical system approach. Journal of Fluid Mechanics 227, 245-272.

Star-ccm+ v. 6.04 user manual. 2011. Cd-Adapco.

Shur, M.L., Spalart, P.R., Strelets, M.Kh., Travin, A.K., 2008. A hybrid RANS-LES approach with delayed-DES and wall-modeled LES capabilities. International Journal of Heat and Fluid Flow 29, 1638-1649.

Viellieber, M., Class, A. G., 2012. Anisotropic porosity formulation of the coarse-grid-CFD (CGCFD). ICONE 20 Annheim, CA USA 


\section{Table1}

Pressure rate of strain tensor coefficients for SSG model

\begin{tabular}{|c|c|c|c|c|c|c|}
\hline Cs1 & Cs2 & Cr1 & Cr2 & Cr3 & Cr3* & Cr4 \\
\hline 1.7 & 1.05 & 0.625 & 0.2 & 0.8 & 0.65 & 0.9 \\
\hline
\end{tabular}

Table 2

Bare grid meshes for source forcing technique

\begin{tabular}{|l|r|}
\hline Bare Grid & N. Elements (millions) \\
\hline Mesh1 & 0.2 \\
\hline Mesh2 & 1 \\
\hline Mesh3 & 6.5 \\
\hline
\end{tabular}


Table 3

Different forcing options analyzed with Code_Saturne ${ }^{1}$

\begin{tabular}{|l|c|c|c|c|c|c|}
\hline $\begin{array}{l}\text { Sources } \\
\text { Forcing }\end{array}$ & $\mathrm{u}$ & $\mathrm{v}$ & $\mathrm{w}$ & $\mathrm{u}_{\mathrm{i}} \mathrm{u}_{\mathrm{j}}$ & $\begin{array}{c}\text { Extension 1Dh above } \\
\text { Vanes }\end{array}$ & $\begin{array}{c}\text { Numerical } \\
\text { Stability }\end{array}$ \\
\hline Option 1 & $\mathrm{x}$ & $\mathrm{x}$ & $\mathrm{x}$ & $\backslash$ & $\backslash$ & Stable \\
\hline Option 2 & $\mathrm{x}$ & $\mathrm{x}$ & $\backslash$ & $\backslash$ & $\backslash$ & Stable \\
\hline Option 3 & $\mathrm{x}$ & $\mathrm{x}$ & $\mathrm{x}$ & $\mathrm{x}$ & $\backslash$ & Stable \\
\hline Option 4 & $\mathrm{x}$ & $\mathrm{x}$ & $\backslash$ & $\mathrm{x}$ & $\mathrm{x}$ & Stable \\
\hline Option 5 & $\mathrm{x}$ & $\mathrm{x}$ & $\backslash$ & $\mathrm{x}$ & Stable \\
\hline
\end{tabular}

Table 4

Different forcing options analyzed with Star-ccm $+^{2}$

\begin{tabular}{|l|c|c|c|c|c|c|}
\hline $\begin{array}{l}\text { Sources } \\
\text { Forcing }\end{array}$ & $\mathrm{u}$ & $\mathrm{v}$ & $\mathrm{w}$ & $\mathrm{u}_{\mathrm{i}} \mathrm{u}_{\mathrm{j}}$ & $\begin{array}{c}\text { Extension 1Dh above } \\
\text { Vanes }\end{array}$ & $\begin{array}{c}\text { Numerical } \\
\text { Stability }\end{array}$ \\
\hline Option 1 & $\mathrm{x}$ & $\mathrm{x}$ & $\mathrm{x}$ & $\backslash$ & $\backslash$ & Unstable \\
\hline Option 2 & $\mathrm{x}$ & $\mathrm{x}$ & $\backslash$ & $\backslash$ & $\backslash$ & Stable \\
\hline Option 3 & $\mathrm{x}$ & $\mathrm{x}$ & $\mathrm{x}$ & $\mathrm{x}$ & $\backslash$ & Unstable \\
\hline Option 4 & $\mathrm{x}$ & $\mathrm{x}$ & $\backslash$ & $\mathrm{x}$ & $\mathrm{x}$ & Stable \\
\hline Option 5 & $\mathrm{x}$ & $\mathrm{x}$ & $\backslash$ & $\mathrm{x}$ & Stable \\
\hline
\end{tabular}



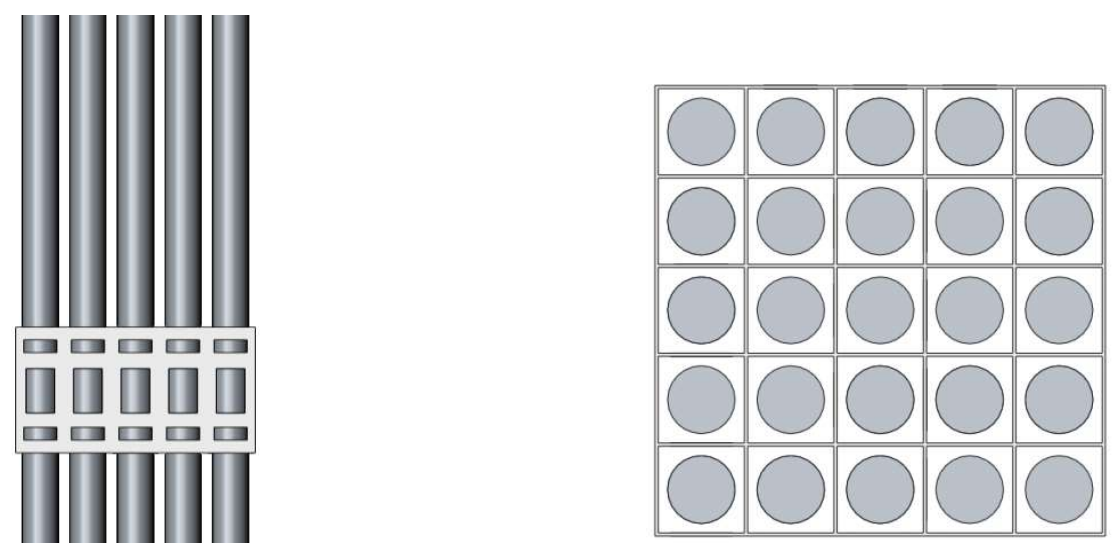

Fig. $1 a, b$ From the left side and top view of the rod bundle and bare walls spacer. All the complex geometrical features were removed.

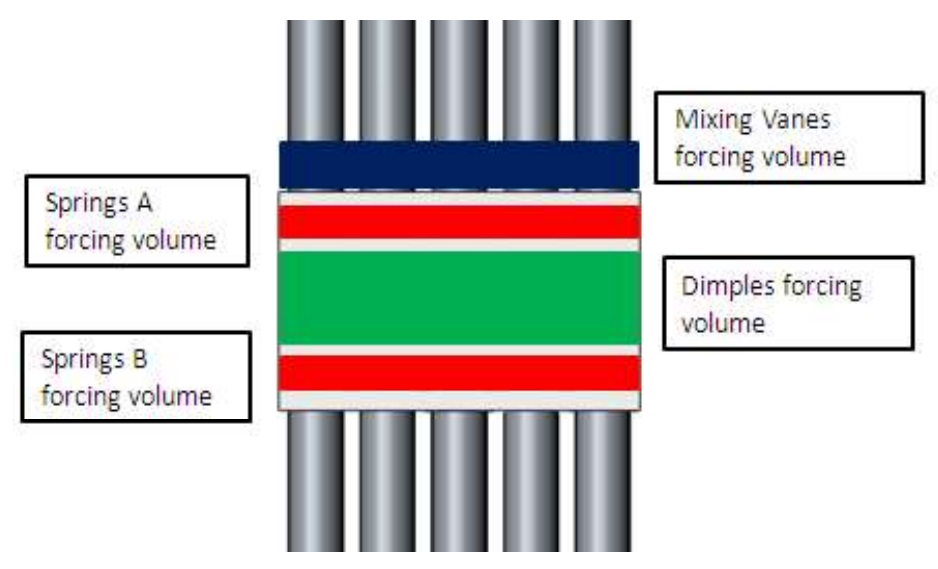

Fig. 2 Four volumes used for solution extraction for body fitted mesh calculation and source forcing for bare grid ones. 
Body Fitted Mesh
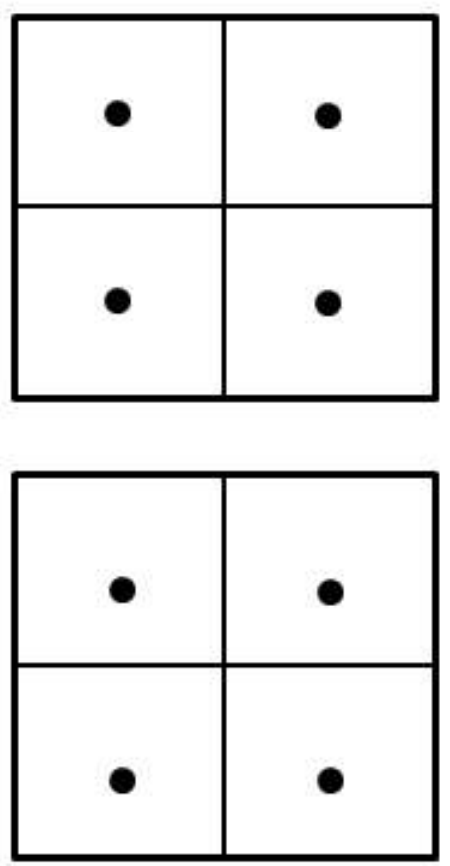

Coarser Bare Grid Domain Mesh nodes are used to extrapolate the values to be imposed in the corresponding one

\section{Extrapolation}
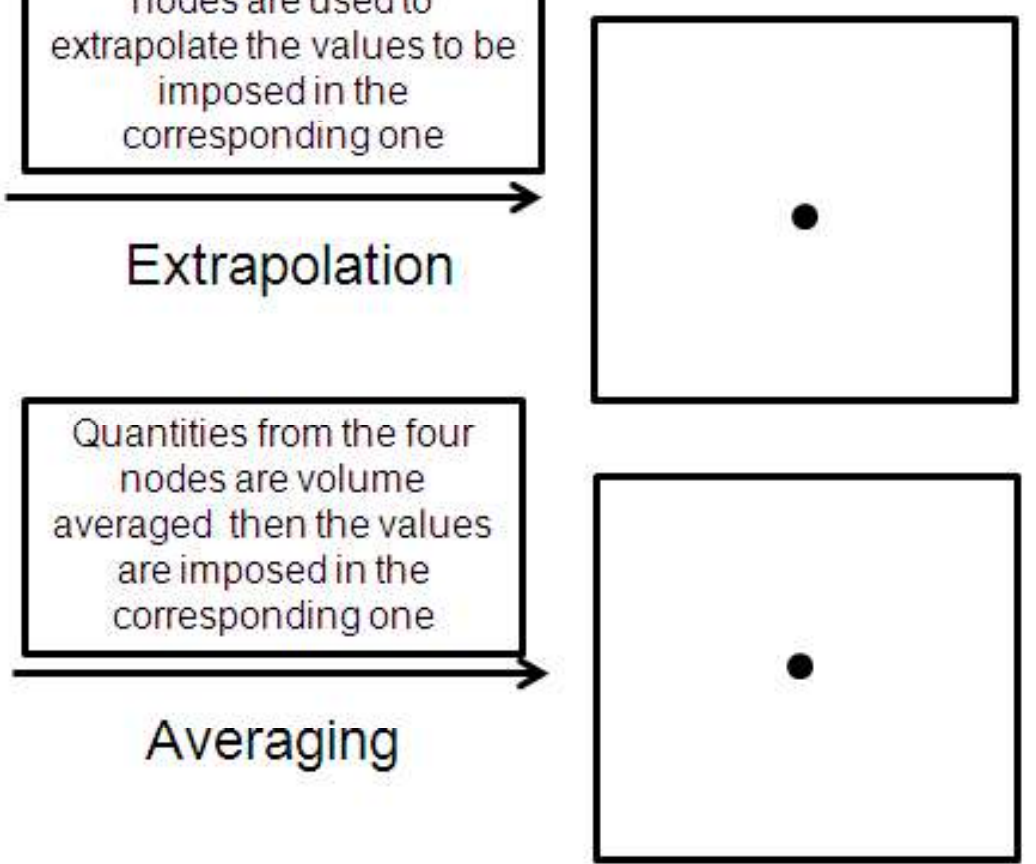

Fig.3 Comparison between the extrapolation and averaging technique to definition velocities and Reynolds stresses in the coarse bare grid domain 


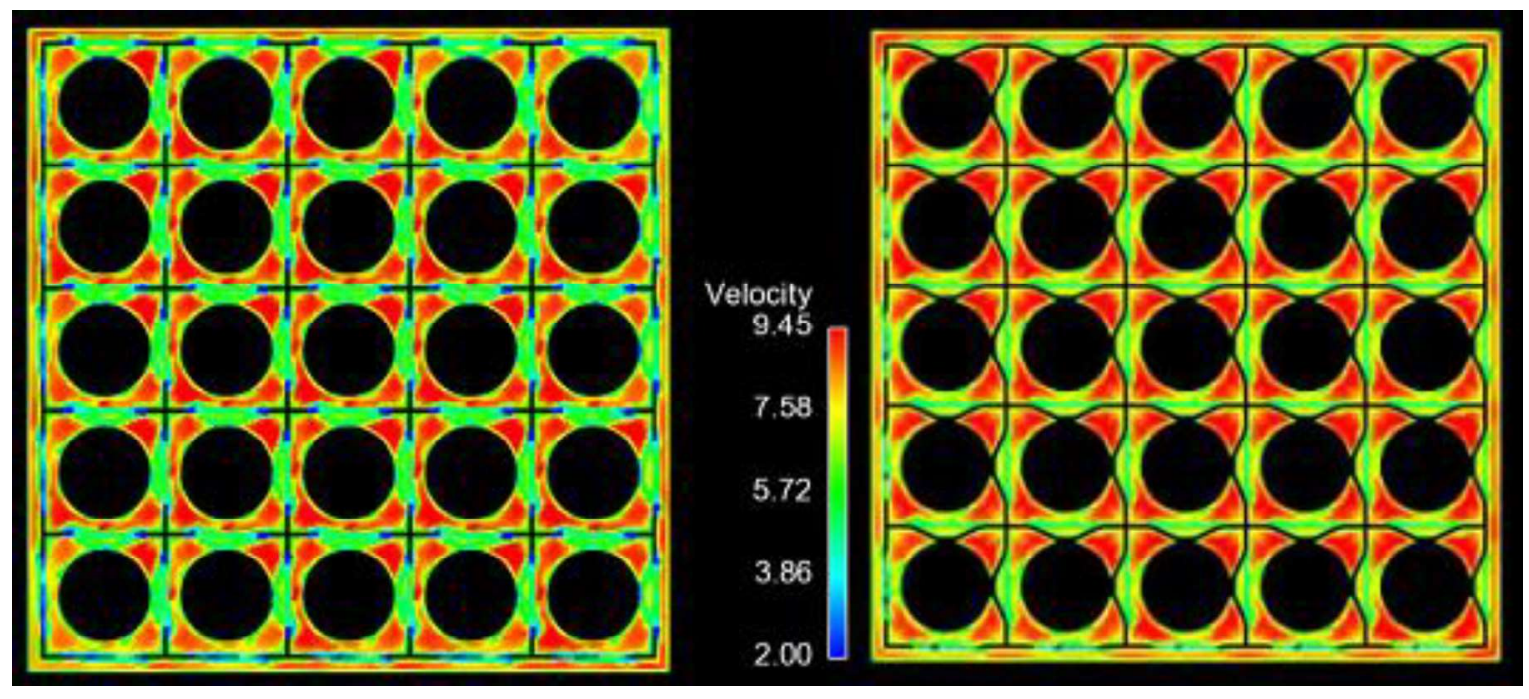

Fig. 4 Left: cross sectional view of the bare grid computational domain at the spring $A$ axial position; around each pin the shadow generated by the forcing solution as the springs were represented in the mesh. Right: cross sectional view of the body-fitted mesh where the presence of springs is clearly visible

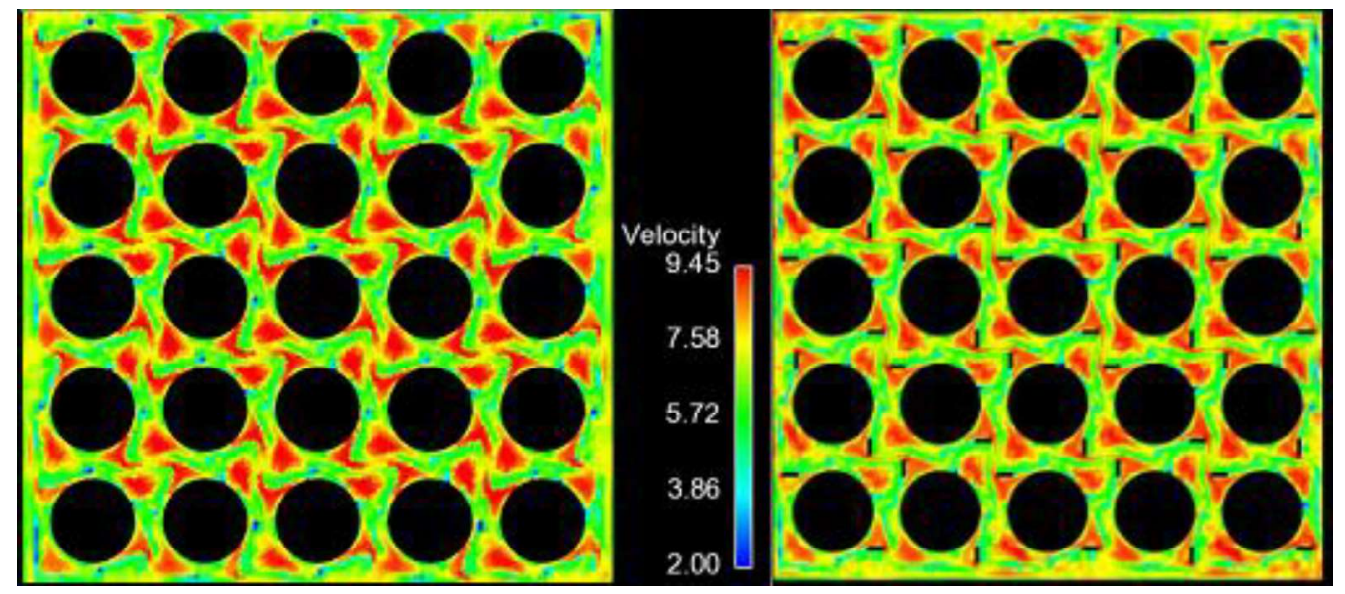

Fig. 5 Left: cross sectional view of the bare grid computational domain at the mixing vanes axial position; around each pin the shadow generated by the forcing solution as the vanes were represented in the mesh. Right: cross sectional view of the body-fitted mesh where the presence of mixing vanes is clearly visible 

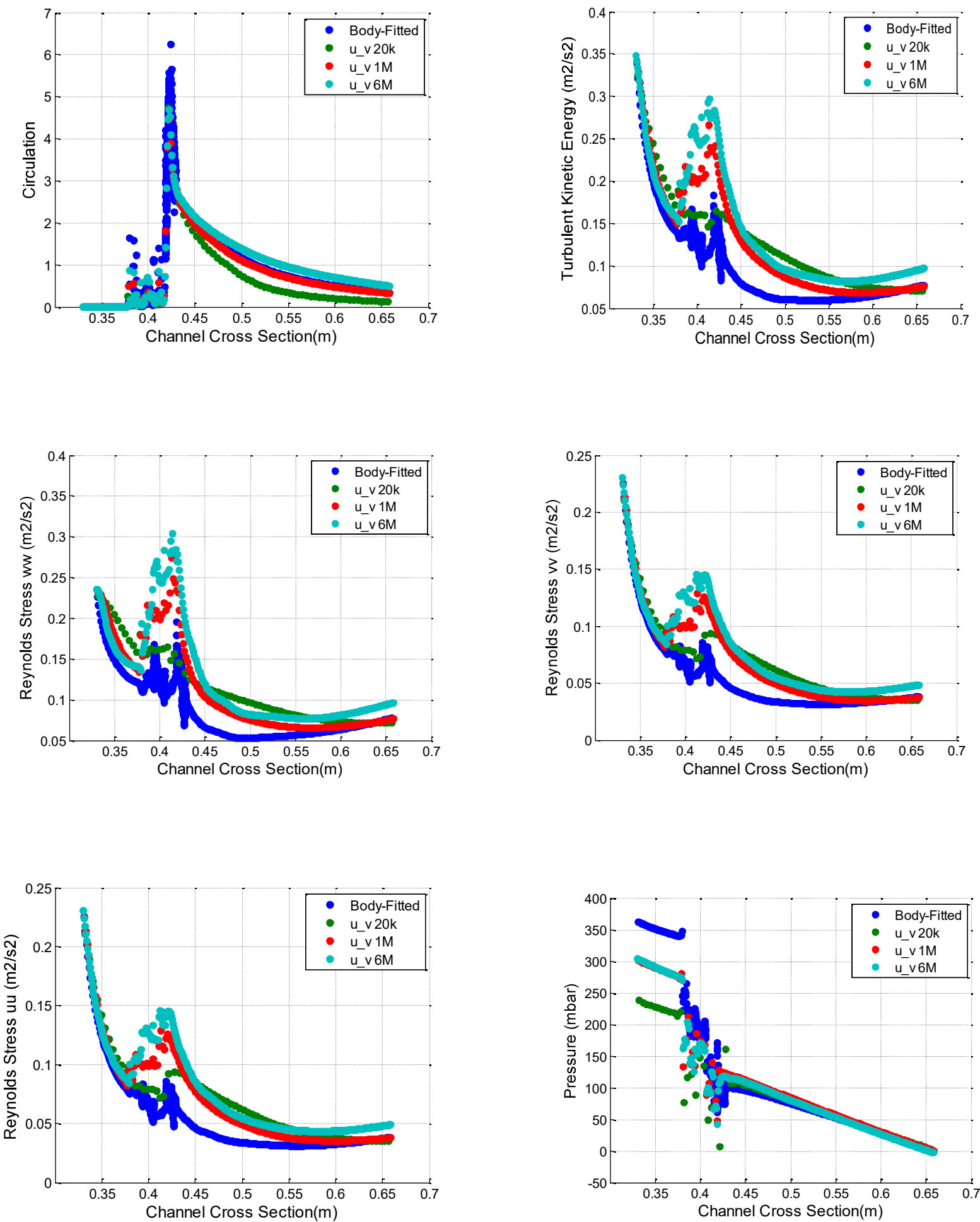

Fig. 6 a,b,c,d,e,f. Integral quantities evolution along the axial direction with forcing of tangentials velocity components using SSG model using implicit forcing 

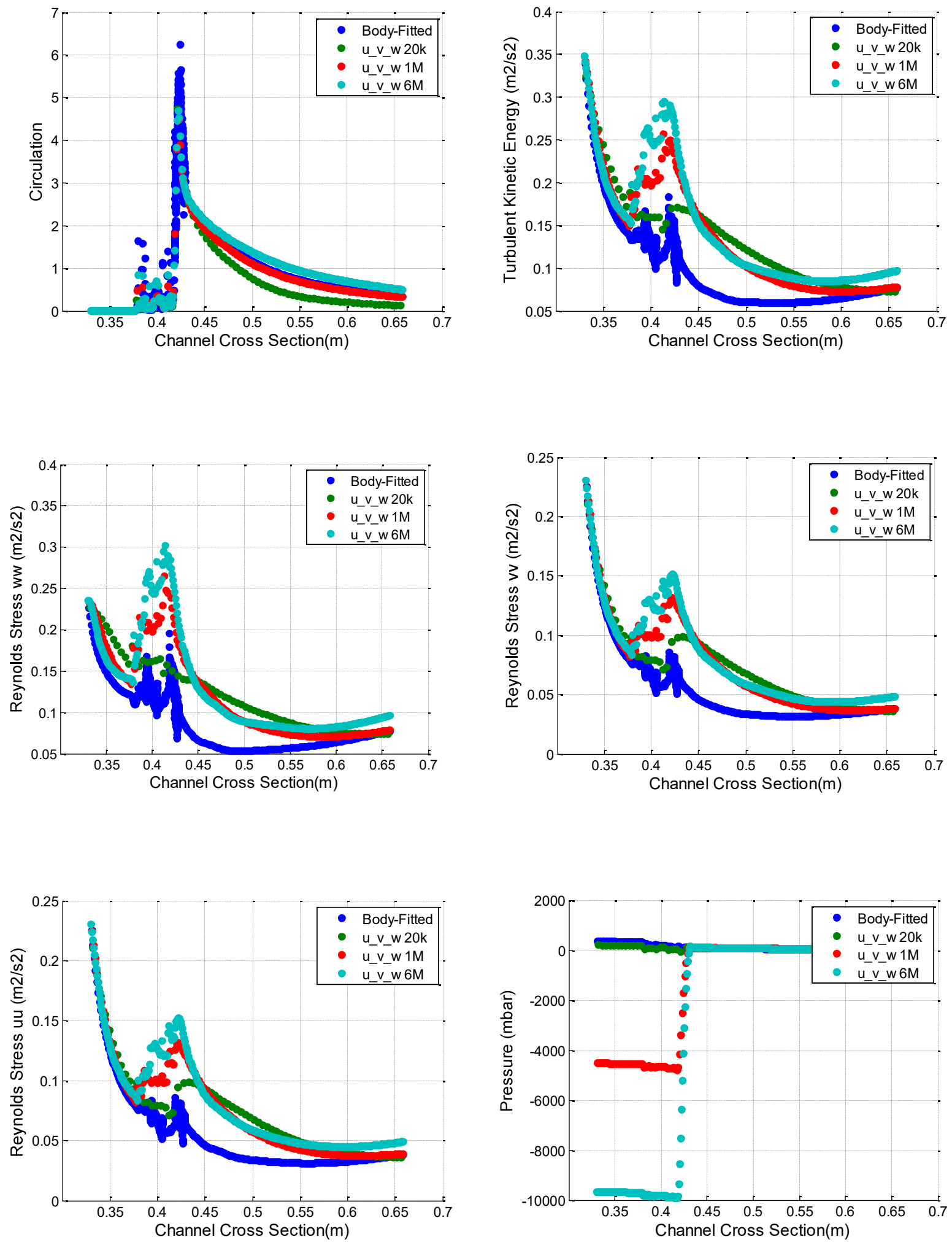

Fig. 7 a,b,c,d,e,f Integral quantities evolution along the axial direction with forcing of tangentials and axial velocity components using SSG model using implicit forcing 

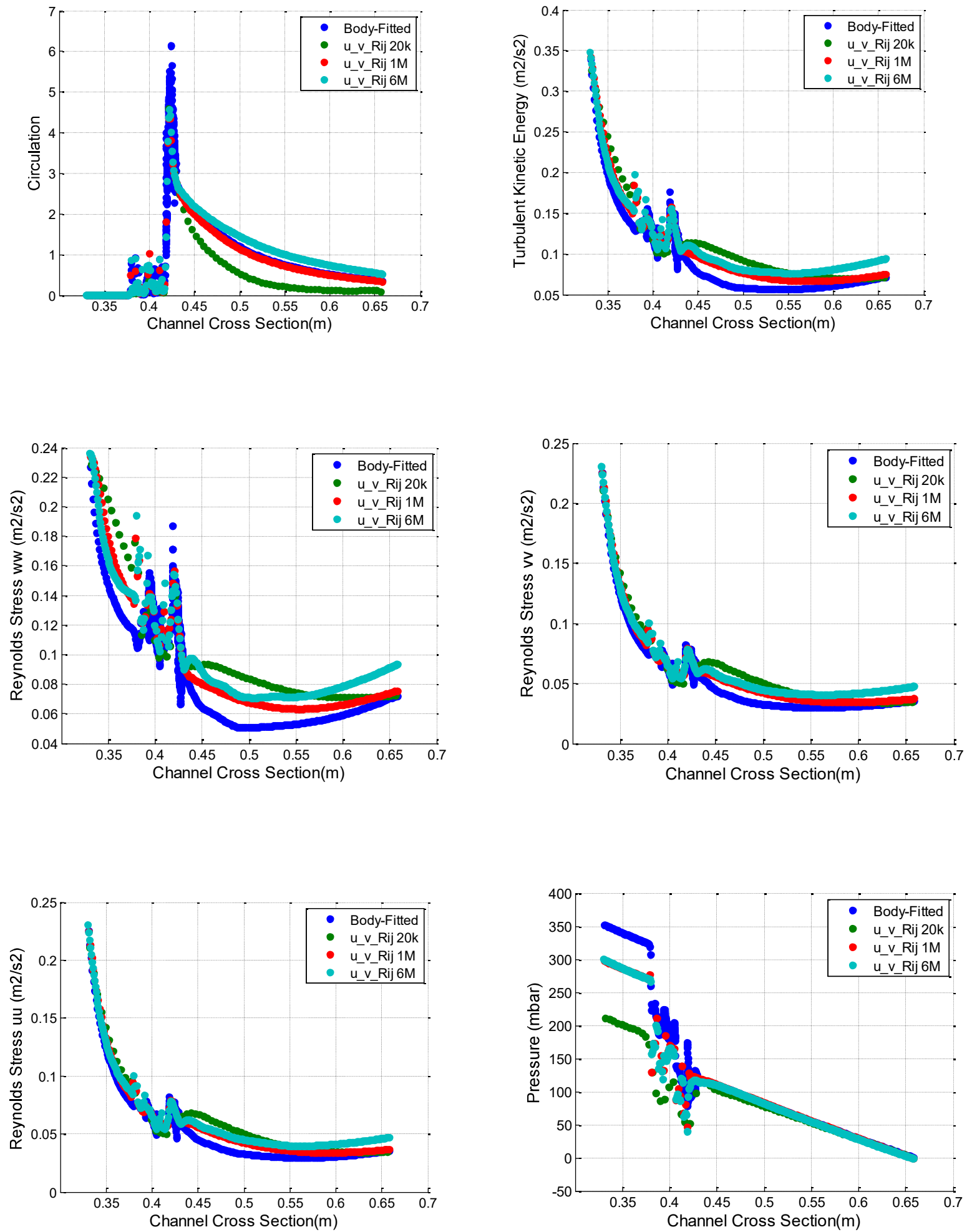

Fig. 8 a,b,c,d,e,f. Integral quantities evolution along the axial direction with forcing of tangentials velocity components and Reynolds stresses using SSG model using implicit forcing 

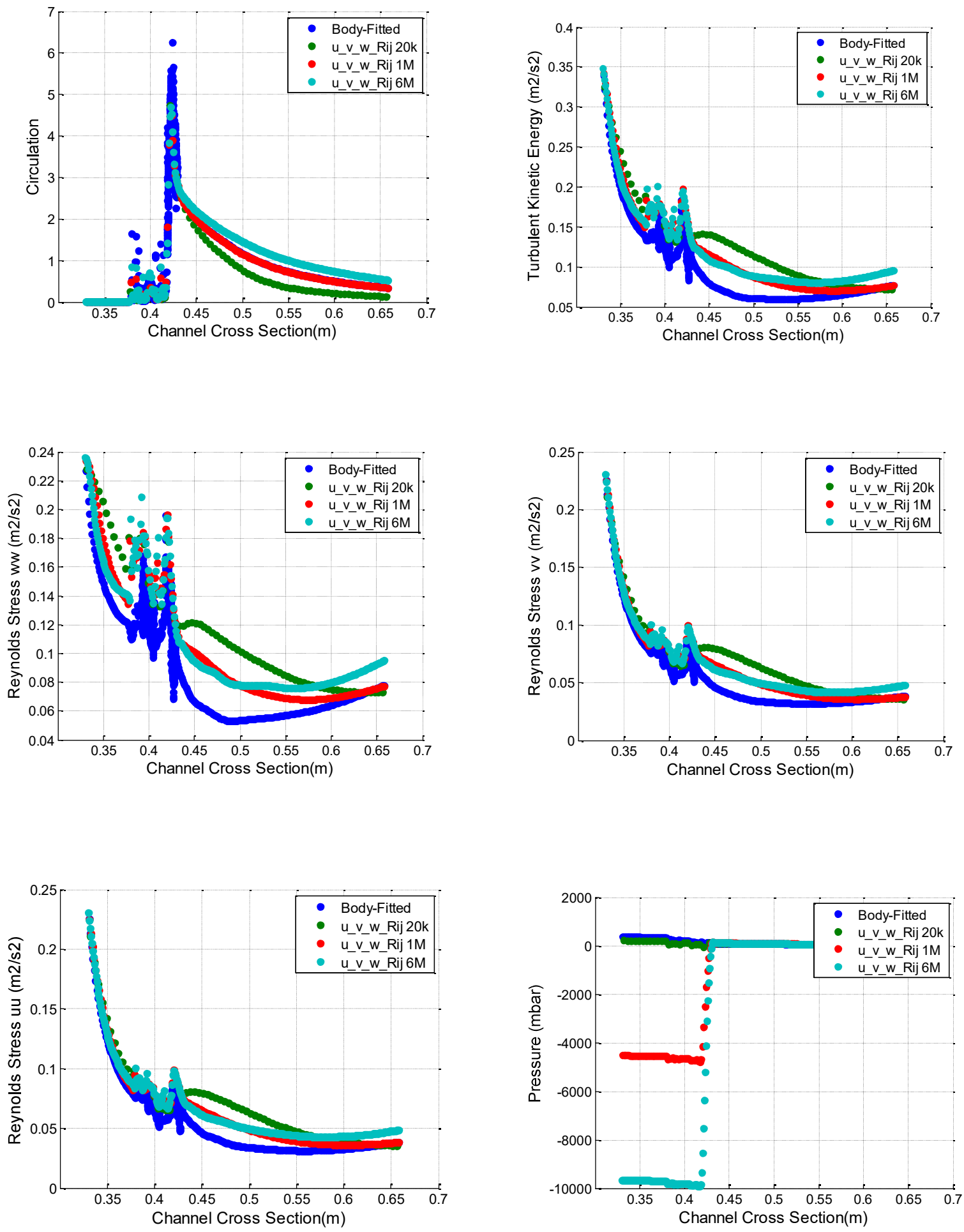

Fig. 9 a,b,c,d,e,f. Integral quantities evolution along the axial direction with forcing of tangentials and axial velocity components and Reynolds stresses using SSG model using implicit forcing 

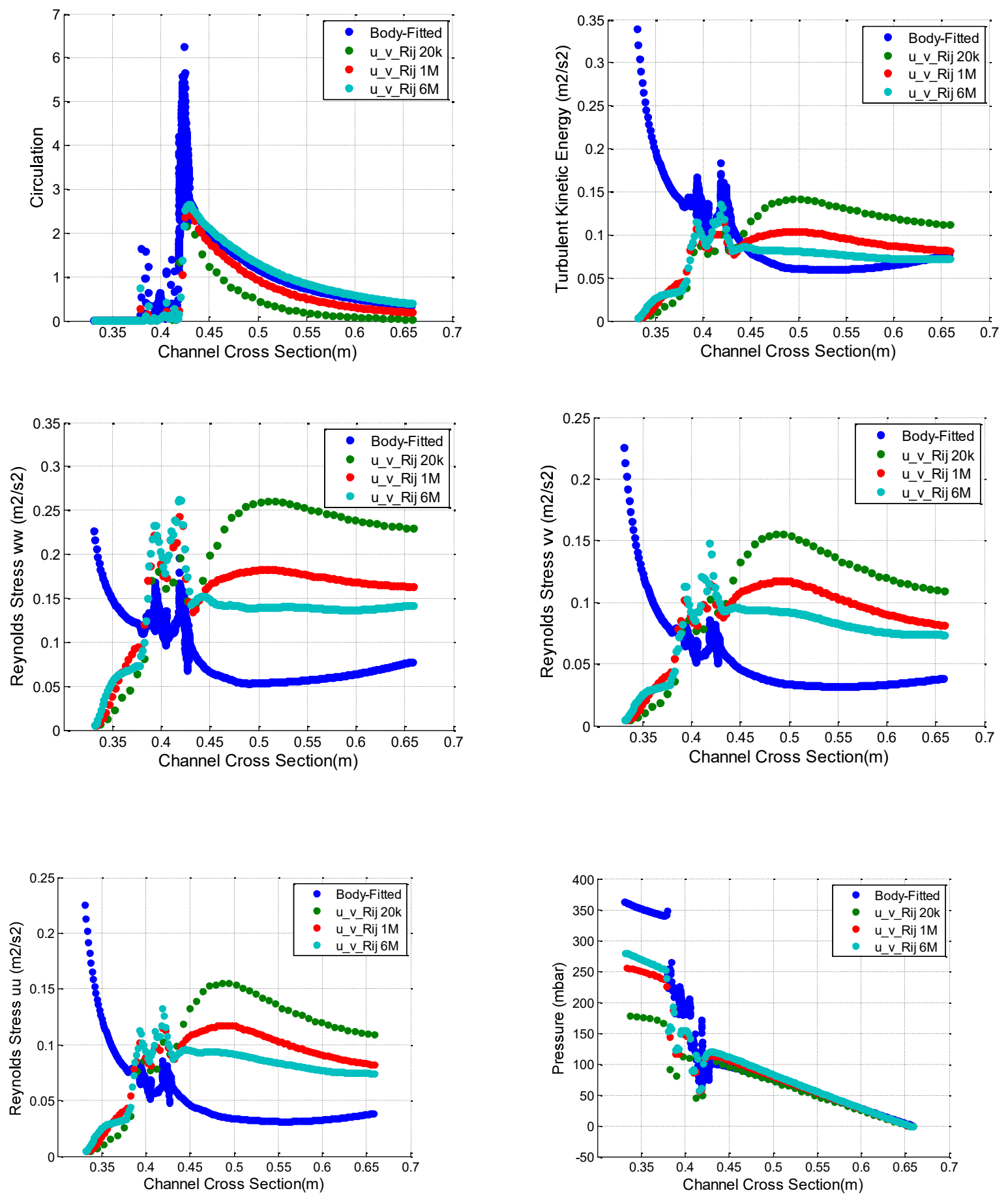

Fig. 10 a,b,c,d,e,f. Integral quantities evolution along the axial direction with forcing of tangentials components and Reynolds stresses using SSG model using explicit forcing 


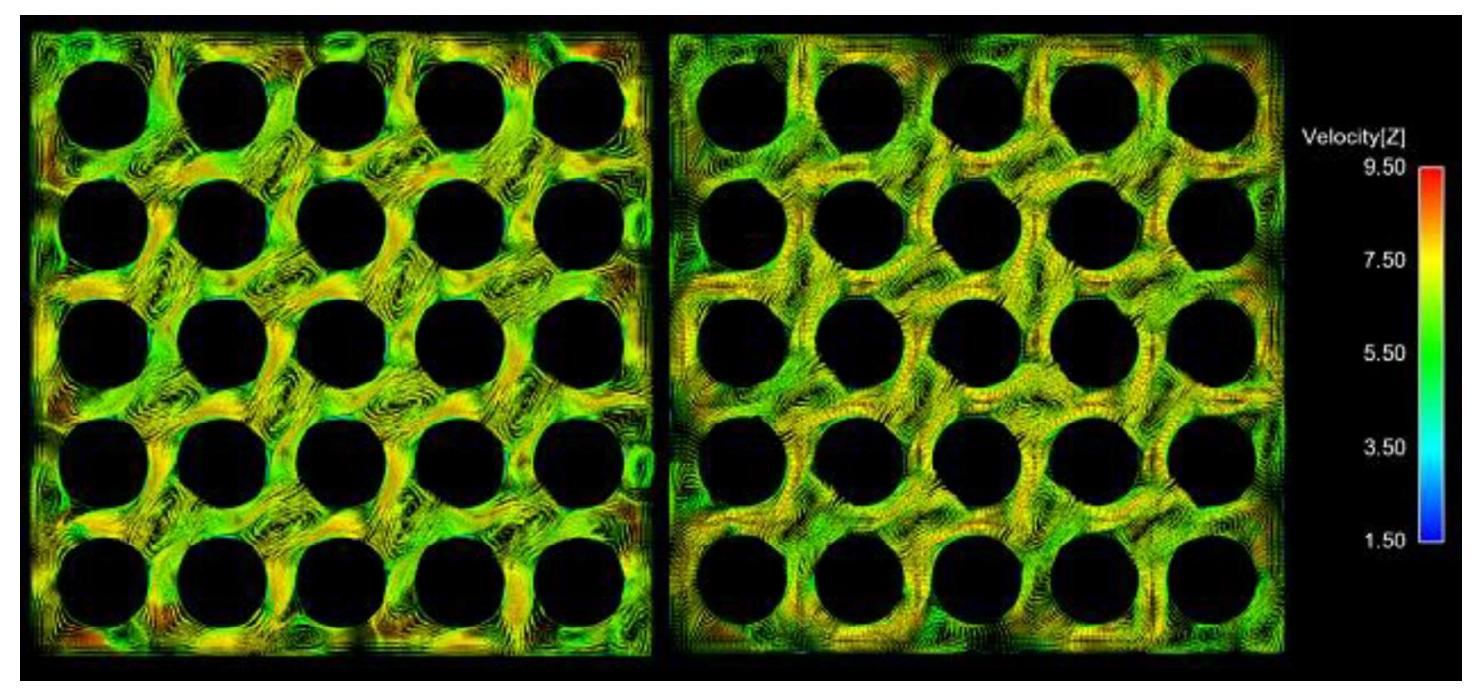

Fig. 11 Left: Body fitted solution Right: 6 million mesh tangential velocity structures $1.5 \mathrm{Dh}$ downstream mixing vanes

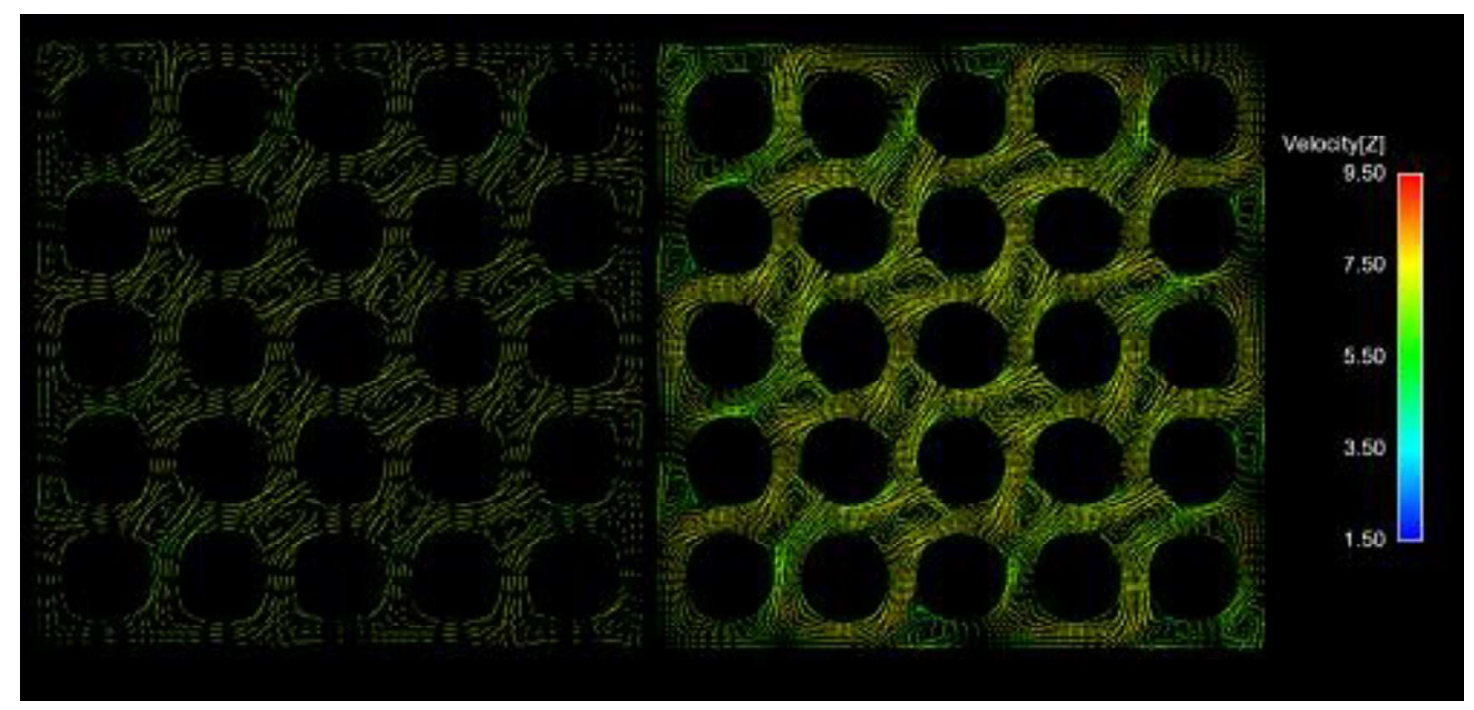

Fig. 12 Left: 20,000 elements mesh Right: 1 million mesh tangential velocity structures $1.5 \mathrm{Dh}$ downstream mixing vanes 


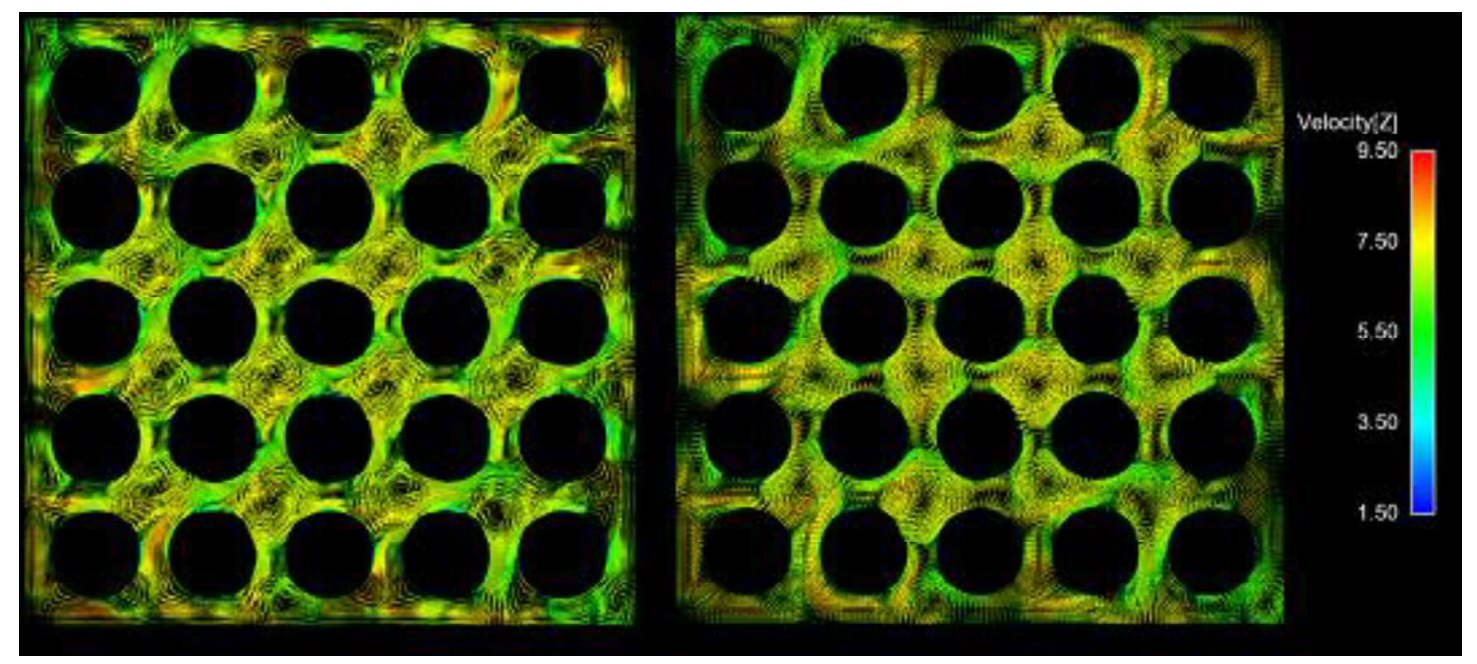

Fig. 13 Left: Body fitted solution Right: 6 million mesh tangential velocity structures 5 Dh downstream mixing vanes

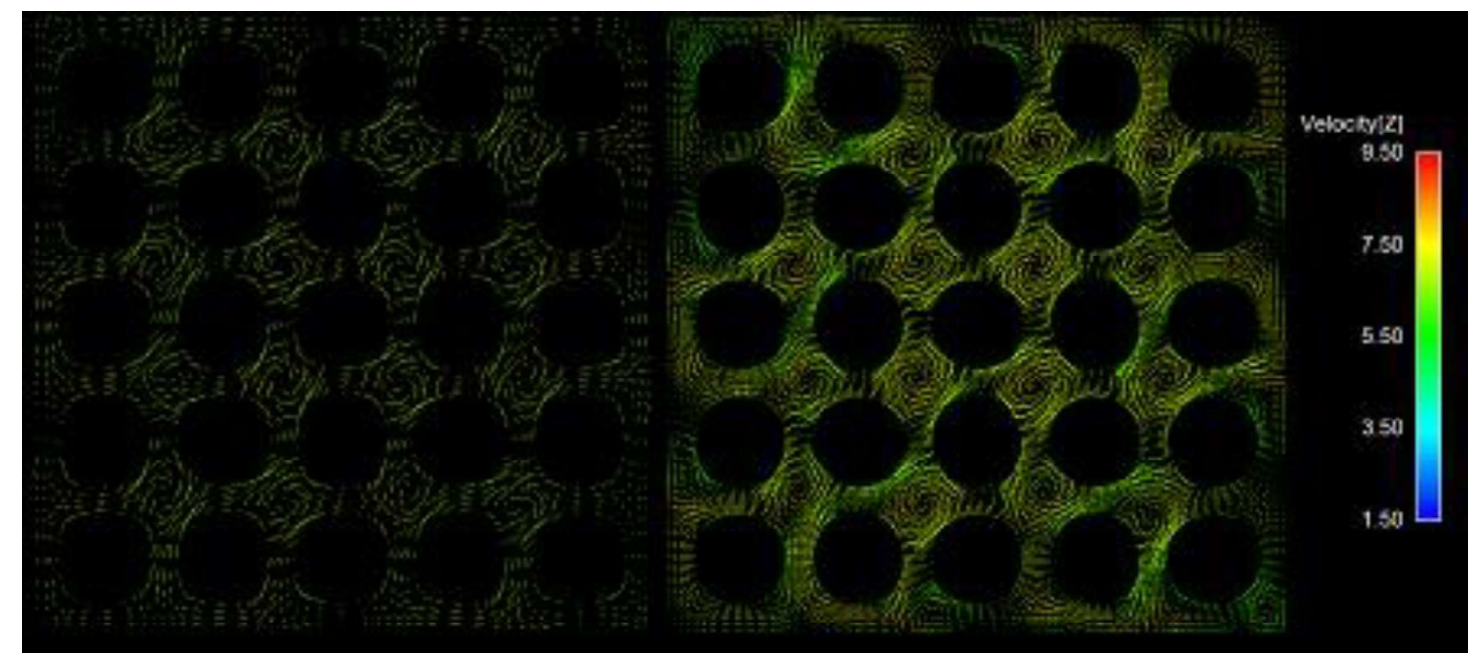

Fig. 14 Left: 20000 elements mesh Right: 1 million mesh tangential velocity structures 5 Dh 


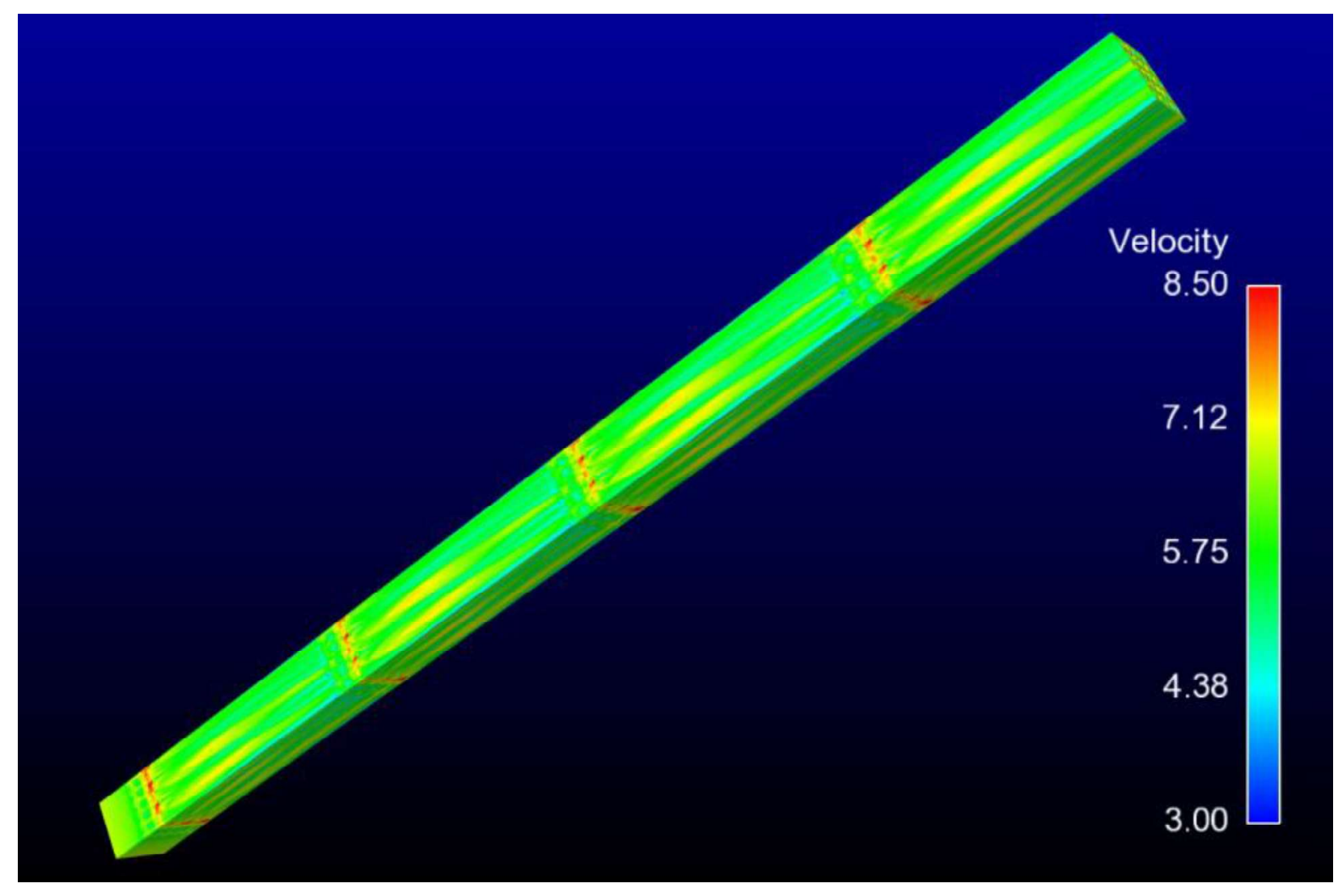

Fig. 15 Calculation with SSG model for four grid spans with a total mesh size of four million elements 